Boletín de la Sociedad Geológica Mexicana

VOLUMEN 61, NÚM. 2, 2009, P. 147-153

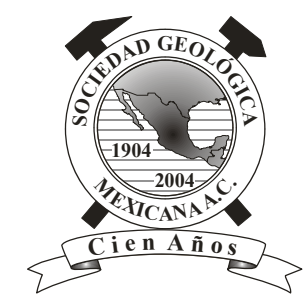

\title{
Los fósiles del Museo de Múzquiz A. C. y su resguardo patrimonial por el Instituto Nacional de Antropología e Historia
}

\author{
Felisa J. Aguilar ${ }^{1, *}$, Héctor Porras-Múzquiz ${ }^{2}$ \\ ${ }^{1}$ Centro INAH Coahuila. Nicolás Bravo Norte 120, Zona Centro, Saltillo, Coahuila, C. P. 25000 \\ ${ }^{2}$ Museo de Múzquiz A. C. Zaragoza 209 Oriente, Centro, Melchor Múzquiz, Coahuila. C. P. 26340 \\ *felisaaguilar@yahoo.com.mx
}

\begin{abstract}
Resumen
La Asociación Civil Museo de Múzquiz se constituyó en febrero de 2005, por aficionados a la paleontología con el objetivo de resguardar y custodiar el patrimonio paleontológico de la región de Múzquiz, Coahuila, además de constituirse en un vínculo entre los profesionales de la disciplina y la sociedad civil para el estudio y la difusión de los resultados más allá del ámbito académico. La colección incluye Bivalvia, Ammonoidea, Crustacea, Chondrichthyes, Osteichthyes, Mosasauridae y una copia del Muzquizopteryx coahuilensis (Pterosauria), 63 son hipotipos y uno es un plastotipo. En 2008, la colección ingresa como bien patrimonial de la nación a través de su inscripción en el Registro Público de Monumentos y Zonas Arqueológicos del Instituto Nacional de Antropología e Historia bajo el número REG. 1680PJ, con un total de 424 cédulas, de ellas 329 amparan 390 fósiles. Así, el Museo de Múzquiz es el custodio oficial y coadyuvante del INAH en la protección de este patrimonio. Su estudio y difusión ya está dando resultados, con lo que se cumple así el propósito para la que fue creada la Asociación Civil Museo de Múzquiz.
\end{abstract}

Palabras clave: Patrimonio paleontológico, Múzquiz, INAH, México.

\begin{abstract}
The Múzquiz Museum Nonprofit Association was established in February 2005 by palaeontology enthusiasts with the aim of safeguarding and preserving the palaeontological heritage of the region of Múzquiz, Coahuila, as well as providing a link between scholars and the general public for study and the publication of results beyond the academic realm. The collection includes Bivalvia, Ammonoidea, Crustacea, Chondrichthyes, Osteichthyes, Mosasauridae, and an artificial mold of Muzquizopteryx coahuilensis (Pterosauria); 63 of these are hypotypes and one is a plastotype. In 2008 this collection was officially recognized as part of the national heritage through its listing in the Public Registry of Archaeological Zones and Monuments of the National Institute of Anthropology and History (INAH) under the number REG. 1680PJ, with 424 descriptive summaries, 329 of which pertain to 390 fossils. The Múzquiz Museum is the official custodian and collaborates with INAH in protecting this heritage. Its study and dissemination of results are already bearing fruit, fulfilling the purpose for which the Múzquiz Museum Nonprofit Association was created.
\end{abstract}

Keywords: Paleontological heritage, Múzquiz, INAH, Mexico. 


\section{Introducción}

El estado de Coahuila de Zaragoza se ha incorporado al ámbito del reconocimiento nacional e internacional debido al patrimonio paleontológico que yace en las rocas y suelos del estado, principalmente el que corresponde a los restos de dinosaurios. Sin embargo, este grupo no es el único presente, existen otros, tanto de animales como de plantas, que nos dan información sobre al menos tres eras geológicas: la Paleozoica, con el Pérmico; la Mesozoica con el Jurásico y el Cretácico, y la Cenozoica con el Paleógeno y el Pleistoceno (Gradstein et al., 2004; Aguilar y Polaco, 2006) (figura 1).

Sin embargo, todavía falta explorar y prospectar en gran parte de la entidad, con el fin de iniciar estudios y/o corroborar el estado en el que se encuentran muchos de los sitios y los materiales ahí presentes ya conocidos. En los últimos años, tanto los profesionales como los aficionados a la disciplina, han incrementado el desarrollo de proyectos de investigación y exploración en los yacimientos, búsquedas, que algunas veces, al hacerse sin tener programas de investigación bien definidos y exploraciones debidamente realizadas están causando la pérdida irremediable de estos materiales junto con la información asociada (AguilarArellano y Polaco-Ramos, 2006).

Aunado a estos eventos, está el hallazgo fortuito de fósiles, principalmente durante la construcción de obras civiles, apertura de caminos, labores agrícolas, explotación de canteras, actividades mineras o en los cauces de los ríos (Aguilar, 2009). Los hallazgos, en algunos casos, son conocidos sólo en la región en donde suceden (Aguilar, 2009), y gracias a la sociedad civil, cuando está consciente del valor de este patrimonio, es posible que parte de estos materiales se rescate y resguarde, ya que al ser los actores sociales directos coadyuvan en la protección y conservación de su patrimonio (Muñoz-Collazos, 2006) y en ocasiones contribuyen con el hallazgo de sitios y fósiles inéditos (Babin, 1992).

Esta es la situación de los habitantes de Melchor Múzquiz y la relación que han establecido con su patrimonio paleontológico, al generarse una asociación civil que está resguardando los fósiles de la región.

El objetivo de este trabajo es mostrar como esta colección paleontológica ingresó al Registro Público de Monumentos y Zonas Arqueológicos del Instituto Nacional de Antropología e Historia (INAH) y el inventario preliminar que se obtuvo al realizar las actividades de registro.

\section{El patrimonio paleontológico del Municipio de Melchor Múzquiz, Coahuila}

El Municipio de Melchor Múzquiz tiene una extensión territorial de $8128 \mathrm{~km}^{2}$, y se localiza en la parte centro-norte del estado de Coahuila de Zaragoza (entre los $27^{\circ} 33^{\prime} 36^{\prime \prime}$ y $28^{\circ} 59^{\prime} 24^{\prime \prime}$ N y entre los $101^{\circ} 14^{\prime} 24^{\prime \prime}$ y $102^{\circ} 48^{\prime} 00^{\prime \prime} \mathrm{O}$ ). Se encuentra dentro de la Provincia fisiográfica de la
Sierra Madre Oriental (SPP, 1983), y es posible encontrar afloramientos de areniscas, lutitas y asociaciones de calizaslutitas y lutitas-areniscas que corresponden al periodo Cretácico (SPP, 1983) (figura 2).

Algunos yacimientos de calizas, lutitas y margas son explotados comercialmente como material de piedra laja, materiales que son utilizados en la construcción de fachadas y pisos de viviendas (figura 3). Durante la extracción de las lajas, ejemplares fósiles, de invertebrados y vertebrados, con excelente preservación se encuentran con frecuencia. Parte de este material es obsequiado, destruido o guardado por los trabajadores de las canteras, otra parte, desgraciadamente, sale junto con la piedra laja, la cual es vendida a particulares y comercios tanto dentro del país como del extranjero.

Además de estos fósiles, en la región también se encuentra madera petrificada (Estrada-Ruiz et al., 2007; 2008), restos de dinosaurios (Kirkland y Hernández, comunicación personal) y proboscidios (González-Zozaya y Ruiz-Martín, 2004).

\section{El Museo de Múzquiz A. C.}

Dada la problemática existente, un grupo de aficionados a la paleontología, que habita en la cabecera municipal de Melchor Múzquiz, constituyeron en febrero de 2005 la Asociación Civil Museo de Múzquiz, con el fin de resguardar y custodiar el patrimonio paleontológico de la región. Al mismo tiempo, al reconocer la importancia de que los materiales deben ser estudiados, y los resultados generados al término de éstos deben ser divulgados, se constituyó como un vínculo entre los profesionales de la disciplina y la sociedad civil.

Desde su fundación hasta la fecha, parte del acervo que resguarda el Museo de Múzquiz A. C., ha sido estudiado, y otra parte está bajo estudio, por investigadores del Instituto de Geología, de la Universidad Nacional Autónoma de México; la Universidad Autónoma del estado de Hidalgo; la Universidad Autónoma de Baja California Sur, y la Southern Methodist University.

\section{EI INAH y los fósiles}

Por Decreto Presidencial, publicado el 13 de enero de 1986 en el Diario Oficial de la Federación, se adiciona a la Ley Federal sobre Monumentos y Zonas Arqueológicos, Artísticos e Históricos el Artículo 28bis, el cual hace referencia a que las disposiciones sobre monumentos y zonas arqueológicos serán aplicables a los vestigios o restos fósiles (Mirambell et al., 1988; INAH, 1995a). Para dar cumplimiento a esta adición se reforma la Ley Orgánica del Instituto Nacional de Antropología e Historia, con lo cual el INAH se convierte en el encargado de proteger, conservar, restaurar, recuperar, promover y difundir este patrimonio (Mirambell et al., 1988; Aguilar-Arellano y 
ESCALA DEL TIEMPO GEOLÓGICO

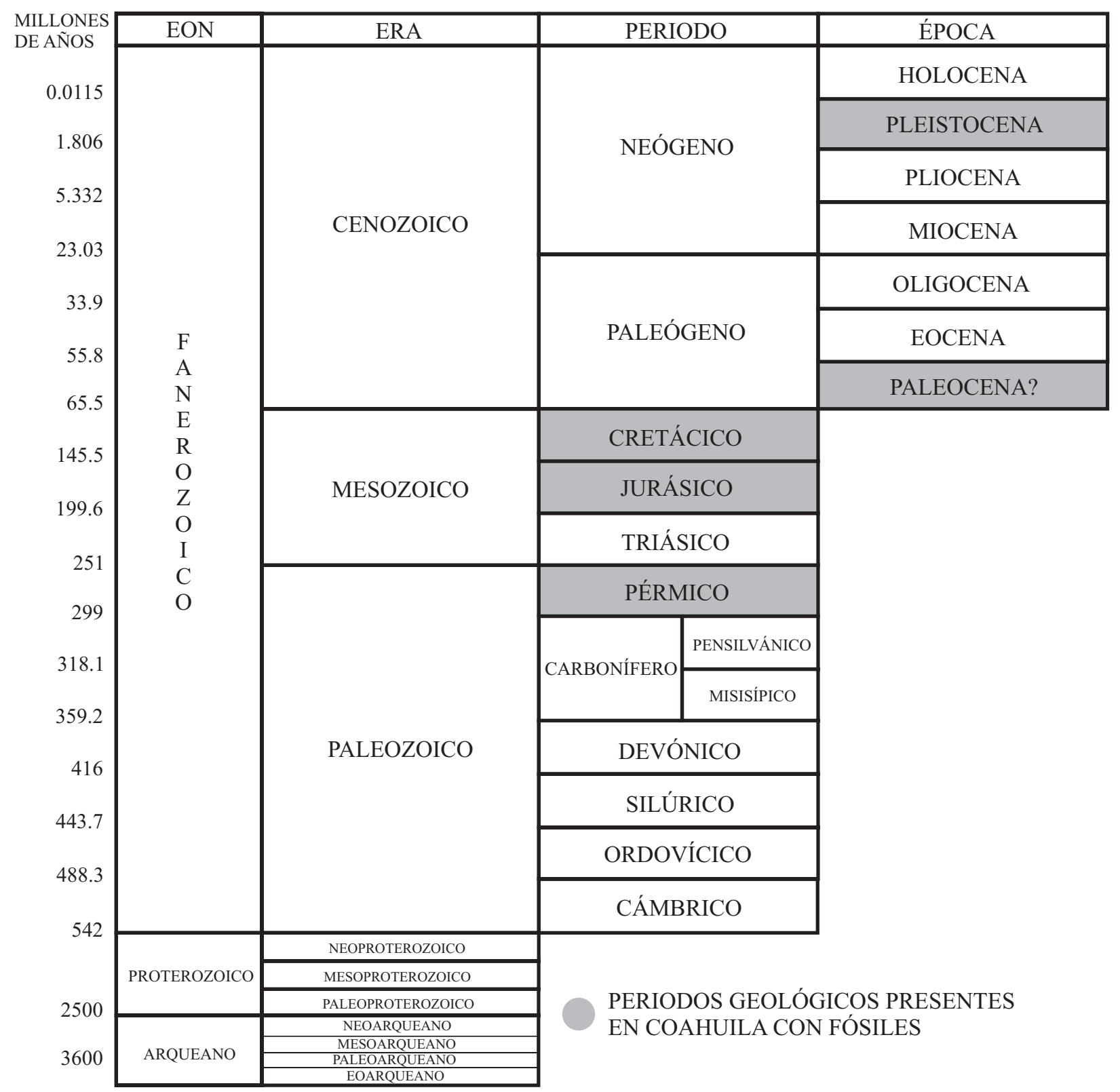

Figura 1. Alcance estratigráfico presente en el estado de Coahuila, la Escala del Tiempo Geológico está de acuerdo a Gradstein et al. (2004).

Polaco-Ramos, 2006). Como parte de esta designación y de acuerdo con el artículo 17 del Reglamento de la Ley Federal sobre Monumentos y Zonas Arqueológicos, Artísticos e Históricos (INAH, 1995b), y considerando que los fósiles y las colecciones de fósiles son bienes nacionales (Ley General de Bienes Nacionales, Artículo VI apartado XVIII), una de las tareas del INAH es llevar a cabo el registro de los bienes muebles paleontológicos que estén en custodia de particulares e instituciones. Aspecto que en el estado de Coahuila a través de la Sección de Paleontología del Centro INAH local se viene realizando desde agosto de 2004.

El INAH a través de la Dirección de Registro Público de Monumentos y Zonas Arqueológicos, ha desarrollado las cédulas que permitan la inscripción de los monumentos arqueológicos y paleontológicos muebles (tabla 1), entre la información que se anota están (INAH, 1995b): la naturaleza del monumento, y el nombre con que se le conozca; la descripción del mueble (ejemplar) y el lugar donde se encuentre, y el nombre y domicilio de quien lo detente.

El trámite de registro de la colección se da a petición de la parte interesada al presentar la forma oficial aprobada y con los datos que ahí se exigen (trámite INAH-00-007; INAH, 2009). Con ello se da un número de inscripción, el cual identifica la colección. Los bienes paleontológicos 


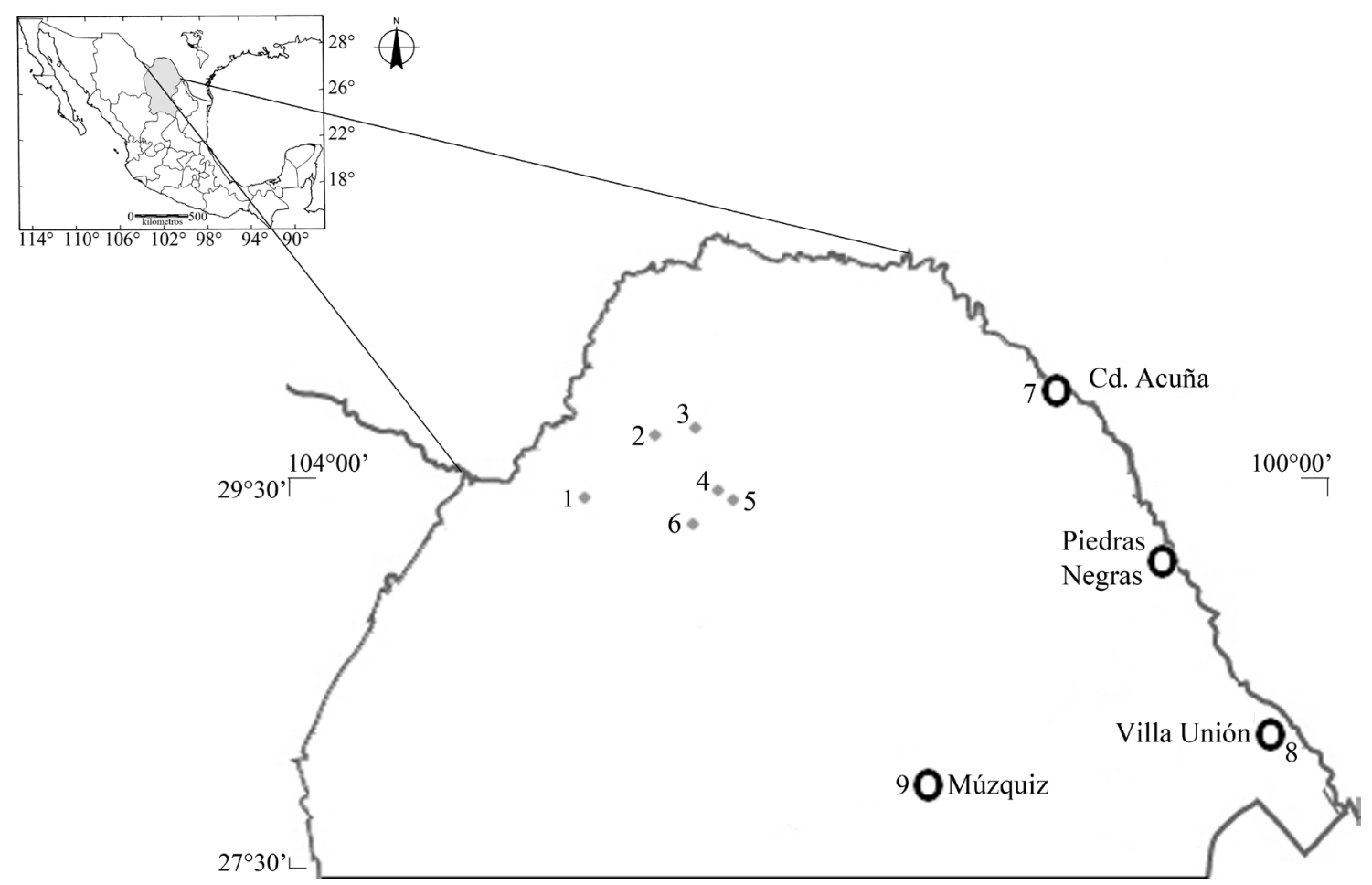

Figura 2. Localización del municipio de Melchor Múzquiz, se marcan las localidades en las que se ha recuperado material paleontológico y que resguarda el Museo de Múzquiz A. C. 1) Jaboncillos, 2) La Mula, 3) Carranza, 4) Los Temporales, 5) La Rosa, 6) El Rosario, 7) Cd. Acuña, 8) Villa Unión y 9) Múzquiz.

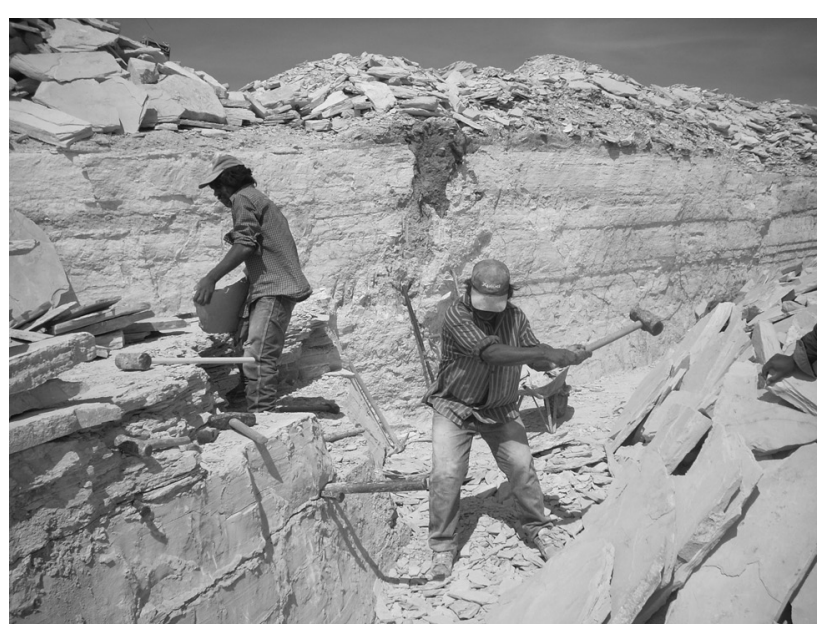

Figura 3. Cantera los Temporales, se observa trabajando a los cantereros

que la integren se identifican con esta sigla, este número lo proporciona la Dirección de Registro Público de Monumentos y Zonas Arqueológicos (INAH, 2003).

Una vez llenadas las cédulas de catálogo para el registro y la inscripción pública se remiten a la Subdirección de Registro de Monumentos Arqueológicos Muebles, donde se ingresará dicha información al Registro Público de Monumentos y Zonas Arqueológicos, y expedirán al interesado la constancia del registro (INAH, 1995b; 2003), quedando la colección inscrita como bien patrimonial de la nación.

\section{El registro de la colección Museo de Múzquiz A. C.}

Con el fin de atender la solicitud de registro de los bienes muebles arqueológicos y paleontológicos de la Asociación Civil Museo de Múzquiz, presentada al INAH a través de su presidente, personal de la Subdirección de Registro de Monumentos Arqueológicos Muebles, para lo arqueológico, y la Sección de Paleontología del Centro INAH Coahuila, para lo paleontológico, se trasladaron al domicilio donde se resguarda la colección (Zaragoza 209 Oriente, Centro, Melchor Múzquiz, Coahuila).

Ahí se tomaron los datos de la cédula correspondiente al tipo de objeto que está en la colección. En el caso de lo paleontológico se tomaron los datos de la cédula (tabla 1) así como las medidas de los ejemplares, el número de catálogo puesto por la Asociación Civil (en este caso ellos designaron la sigla MUZ), y la determinación taxonómica a la que pertenecen los ejemplares. Con el número de registro asignado a la colección por parte de la Subdirección de Registro de Monumentos Arqueológicos Muebles (REG1680PJ), se marcaron las piezas, a las cuales, además, se les asignó un número consecutivo de registro (e. g. REG1680PJ 96). Para el marcado se utilizó una base de pintura de acrílico blanca, el número se colocó con tinta china negra y posteriormente se colocó una capa de barniz 
Tabla 1. Información incluida en la cédula de registro correspondiente a los fósiles, se da una pequeña descripción de lo que se requisita en cada rubro.

Rubro

Definición
Es el número que le corresponde a la pieza que se está describiendo, se designa de manera consecutiva.

Es el nombre de la persona física o institución que solicitó el registro. Se consigna tal como se presentó en la solicitud.

Domicilio en donde se ubica la colección.

El que se le haya otorgado por la Dirección de Registro Público de Monumentos y Zonas Arqueológicos.

Persona que dictamine, registre, fotografíe y llene la cédula.

El día que se llenó la cédula.

Si se trata de un objeto unitario, un lote o un objeto compuesto.

Directa o indirecta.

Clasificación taxonómica, al nivel al que se pueda llegar.

Era, periodo y época, así como la formación geológica.

En el caso del lote se toma tanto la del objeto de menor tamaño como el de mayor tamaño.

Si está completo o incompleto, si es original o reciente (se refiere a que el ejemplar se trata de un duplicado).

Buen estado, restaurado, erosionado, etc.

Cuadro, bodega, a la intemperie, etc.

De donde fue extraído el ejemplar o recolectado.

En este caso si los ejemplares tienen otros números o leyendas marcados.

Todo lo que se considera relevante del ejemplar y que no haya sido considerado en la cédula. transparente (figura 4). Cada pieza se fotografió con una cámara digital con una resolución de 300 dpi y un tamaño de 1280x960 pixeles y colocándole una escala de comparación.

Algunos de los ejemplares han servido para ilustrar publicaciones o bien formado parte de estudios, para lo cual se agregó la referencia bibliográfica correspondiente en el apartado de observaciones, y es recomendable anexar al expediente un sobretiro del artículo o en su defecto una copia del mismo.

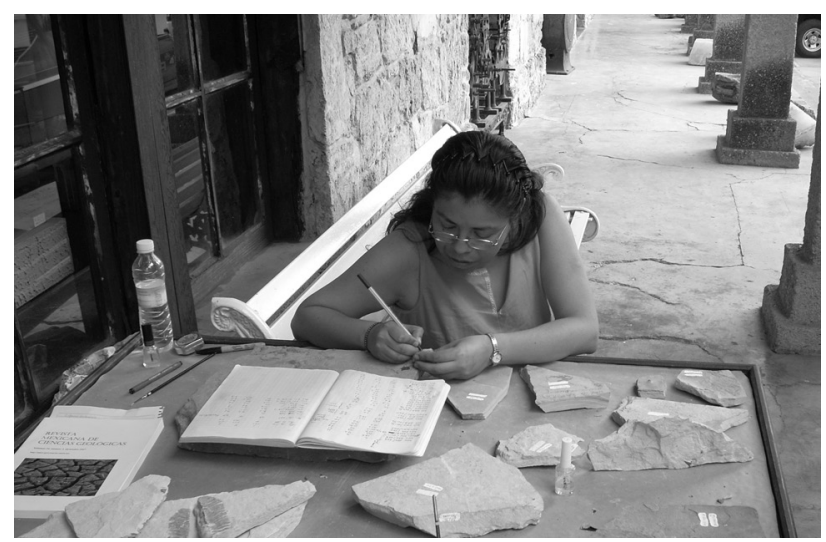

Figura 4. Actividades realizadas durante el registro de la colección paleontológica, en el domicilio donde se resguarda el material.

\section{Resultados}

El acervo del Museo de Múzquiz A. C., está registrado con el número REG1680PJ comprende 424 cédulas. Por las características de los ejemplares, algunos de ellos quedaron registrados en la misma cédula, por lo que 390 ejemplares fósiles están inscritos en 329 cédulas, 95 cédulas corresponden a material arqueológico.

Los ejemplares están en lajas de caliza y margas del Cretácico Tardío (al menos 93.5 millones de años) procedentes de los Grupos Austin y Eagle Ford (BlancoPiñón et al., 2004; Nyborg et al., 2005; Stinnesbeck et al., 2005; Alvarado-Ortega et al., 2006; Vega et al., 2007), que afloran en esta región del estado de Coahuila (Vega et al., 2007).

Los taxa fósiles que se encuentran representados en la colección incluye almejas (Bivalvia), amonites (Ammonoidea), cangrejos, percebes, langostas (Crustacea), tiburones (Chondrichthyes), peces (Osteichthyes), mosasaurios (Mosasauridae) y una copia del Muzquizopteryx coahuilensis (Pterosauria) (Frey et al., 2006) (tabla 2, figuras 5-7). La fauna más abundante son los peces seguidos por los cangrejos, los dientes de tiburón, los menos abundantes son los mosasaurios y los pterosaurios.

En la colección se resguardan 63 hipotipos (espécimen, que no es el tipo, y en el que una descripción o figura suplementaria está basada [Evenhuis, 2008]) de cangrejos (Vega et al., 2007; Guinot et al., 2008), los cuales sirvieron 
Tabla 2. Clasificación taxonómica de los organismos fósiles representados en la Colección del Museo de Múzquiz A. C.

\begin{tabular}{|c|}
\hline Reino Animalia \\
\hline Phylum Mollusca \\
\hline Clase Bivalvia \\
\hline Clase Cephalopoda \\
\hline Phylum Arthropoda ${ }^{1}$ \\
\hline Subphylum Crustacea \\
\hline Clase Maxillopoda \\
\hline Orden Lepadomorpha \\
\hline Familia Stramentidae \\
\hline Género Stramentum \\
\hline Stramentum sp. \\
\hline Clase Malacostraca \\
\hline Orden Stomatopoda \\
\hline Familia Pseudosculdidae \\
\hline Orden Decapoda \\
\hline Familia Nephropidae \\
\hline Subfamilia Nephropinae \\
\hline Familia Palinura \\
\hline Familia Gourretiidae \\
\hline Género Gourretia \\
\hline Gourretia aquilae \\
\hline Familia Cenomanocarcinidae \\
\hline Género Cenomanocarcinus \\
\hline Cenomanocarcinus vanstraeleni (Figura 5) \\
\hline Familia Raninidae \\
\hline Género Cretacoranina \\
\hline Cretacoranina $\mathrm{cf}$. C. dichorus \\
\hline Phylum Chordata ${ }^{2}$ \\
\hline Clase Chondrychthyes \\
\hline Clase Osteichthyes \\
\hline Clase Sauropsida \\
\hline Orden Testudines \\
\hline Orden Squamata \\
\hline Familia Mosasauridae \\
\hline Orden Pterosauria \\
\hline Familia Pteranodontidae \\
\hline Género Muzquizopteryx \\
\hline Muzquizopteryx coahuilensis (Figura 7) \\
\hline
\end{tabular}

${ }^{1}$ Con base en los trabajos de Vega et al. (2007) y Guinot et al. (2008).

${ }^{2}$ Con base en el trabajo de Benton (2004).

de base para describir las características del taxón que representan; además de una copia del original del Muzquizopteryx coahuilensis (figura 7), el cual se considera como plastotipo (un molde artificial elaborado directamente a partir del tipo [Evenhuis, 2008]), y que son tan útiles como los ejemplares que representan (Schenk y McMasters, 1948; Arroyo-Cabrales y Polaco, 2005).

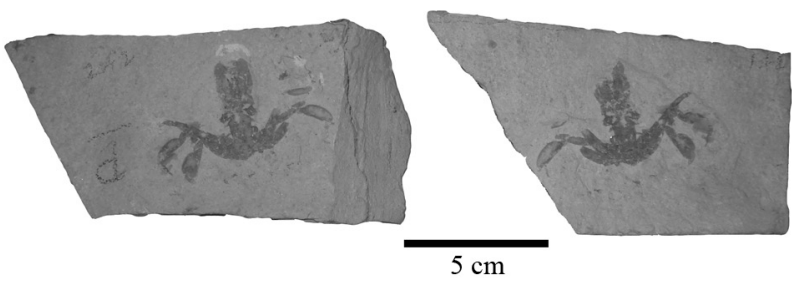

Figura 5. Hipotipo de Cenomanocarcinus vanstraeleni (REG1680PJ237 [MUZ 211]).

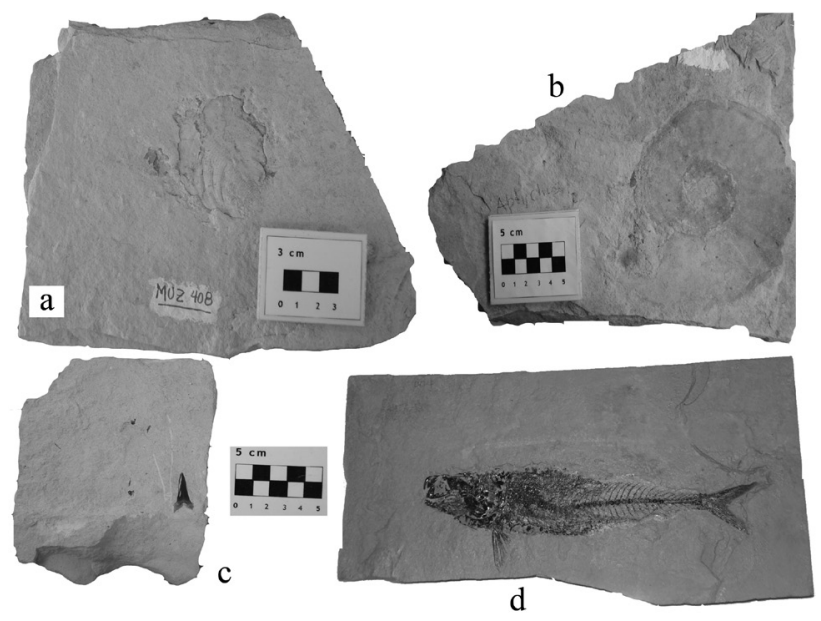

Figura 6. Diversidad de organismos presentes en la colección paleontológica: a) bivalvos, b) amonites, c) condrictios, y d) peces.

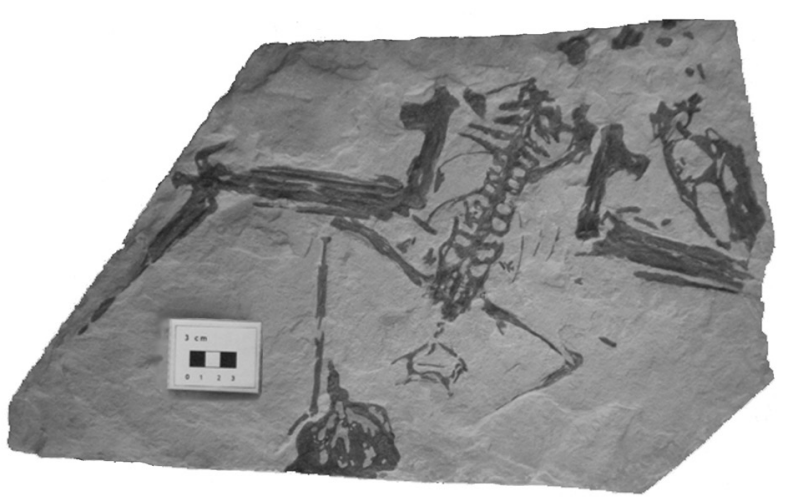

Figura 7. Plastotipo de Muzquizopterix coahuilensis (REG1680PJ376).

Si bien la colección ya ha sido registrada se ignora aún el valor científico potencial que ésta tenga dado que todavía se debe de limpiar, preparar y estudiar parte de las lajas. A pesar de esta ausencia al estar registrado este patrimonio permite pensar en su conservación a través del tiempo y por ende su disponibilidad para estudios futuros. 


\section{Conclusión}

El Museo de Múzquiz A. C. al haber solicitado el registro de la colección, se convierte en el custodio oficial y coadyuvante del INAH en la protección de este patrimonio. Su estudio y difusión ya está dando resultados con dos trabajos publicados y un tercero en preparación por los especialistas que se han acercado a esta colección; lo que cumple así con el propósito para la que fue creada esta asociación. Además, de que involucrará a la comunidad en la protección de este patrimonio a través de las actividades de difusión que realice.

\section{Agradecimientos}

Se agradece a la Dra. María del Carmen Perrilliat, del Instituto de Geología, UNAM; al Prof. Oscar J. Polaco, de la Subdirección de Laboratorios y Apoyo Académico, INAH, y a un revisor anónimo por las observaciones y sugerencias que enriquecieron este escrito.

\section{Referencias}

Aguilar, F.J., 2009, La exposición itinerante "Al rescate del patrimonio paleontológico mexicano": CamINAH por los Centros, experiencias de éxito, 2, 43-47.

Aguilar, F.J., Polaco, O.J., 2006, La protección del patrimonio paleontológico en Coahuila: Saltillo, Coahuila, Instituto Nacional de Antropología e Historia.

Aguilar-Arellano, F.J., Polaco-Ramos, O.J., 2006, E1 patrimonio paleontológico del norte de México, una propuesta para su protección, en: Memorias del segundo encuentro regional para el estudio del patrimonio cultural: Monterrey, Nuevo León, CONARTE y Gobierno del estado de Nuevo León, 88-91.

Alvarado-Ortega, J., Blanco-Piñón, A., Porras-Múzquiz, H., 2006, Primer registro de Saurodon (Teleostei: Ichthyodectiformes) en la cantera La Mula, Estado de Coahuila, México: Revista Mexicana de Ciencias Geológicas, 23, 107-112.

Arroyo-Cabrales, J.. Polaco, O.J., 2005, Ejemplares tipo depositados en la colección paleontológica del Instituto Nacional de Antropología e Historia, en Sánchez-Cordero, V., Medellín, R.A. (eds.), Contribuciones mastozoológicas en homenaje a Bernardo Villa: México, Instituto de Biología, UNAM; Instituto de Ecología, UNAM y CONABIO, 27-37.

Babin, C., 1992, Los yacimientos de fósiles, su protección y su interés cultural en algunos países de Europa, en Fernández López, S. (coord.), Conferencias de la reunión de tafonomía y de fosilización: Madrid, Editorial Complutense, 45-62.

Benton, M.J., 2004, Vertebrate Palaeontology: London, Blackwell Publishing, 3ra. ed.

Blanco-Piñón, A., Porras-Múzquiz, H., Vega-Vera, F., González-Rodríguez, K.A., Alvarado-Ortega, J., 2004, Múzquiz, Coahuila: a new fossiliferous locality, northern Mexico, en: IX Congreso Nacional de Paleontología, Memorias, Tuxtla Gutiérrez, Chiapas: México, Sociedad Mexicana de Paleontología, 23.

Cámara de Diputados del Honorable Congreso de la Unión, 2004, Ley General de Bienes Nacionales: En línea http://www.cna.gob.mx/ eCNA/Espaniol/MarcoNormativo/Leyes/Ley\%20Genera $\% 20$ de $\% 20$ Bienes $\% 20$ Nacionales.pdf\#search=' ${ }^{\prime}$ ey\%20General $\% 20$ de $\% 20$ Bienes $\% 20$ Nacionales'.

Estrada-Ruiz, E., Martínez-Cabrera, H.I., Cevallos-Ferriz, S.R.S., 2007,
Fossil wood from the late Campanian-early Maastrichtian Olmos Formation, Coahuila, Mexico: Review of Palaeobotany and Palynology, 145, 123-133.

Estrada-Ruiz, E., Upchurch, G. R. y Cevallos-Ferriz, S. R. S., 2008, Flora and climate of the Olmos Formation (Upper Campanian-Lower Maastrichtian), Coahuila, Mexico: A preliminary report: Gulf Coast Association of Geological Societies Transactions, 58, 273-283.

Evenhuis, N.L., 2008, A compendium of Zoological Type Nomenclature: a reference source: USA, Bishop Museum Press, Bishop Museum Technical report 41. Consultado en línea: http://hbs.bishopmuseum. org/publications/pdf/bm-tp41.pdf. Enero 2009.

Frey, E., Buchy, M.C., Stinnesbeck, S., González González, A., di Stefano, A., 2006, Muzquizopteryx coahuilensis n.g., n. sp., a nyctosaurid pterosaur with soft tissue preservation from the Coniacian (Late Cretaceous) of northeast Mexico (Coahuila), Oryctos, 6, 47-66.

González-Zozaya, F., Ruiz-Martín, M.C., 2004, Informe de las actividades realizadas del 13 al 21 de abril del 2004: norte y centro del estado de Coahuila: Saltillo, Coahuila, Centro INAH Coahuila, Informe inédito.

Gradstein, F.M., Ogg, J.G., Smith, A.G., Bleeker, W., Lourens, L.J., 2004, A new Geological Time Scale, with special reference to Precambrian and Neogene: Episodes, 27 (2), 83-100.

Guinot, D., Vega, F.J., Van Bakel, B.W.M., 2008. Cenomanocarcinidae n. fam., a new Cretaceous podotreme family (Crustacea, Decapoda, Brachyura, Raninoidia), with comment son related families: Geodiversitas, 30 (4), 681-719.

Instituto Nacional de Antropología e Historia, 1995a, Ley Federal sobre Monumentos y Zonas Arqueológicos, Artísticos e Históricos: México, Instituto Nacional de Antropología e Historia.

Instituto Nacional de Antropología e Historia, 1995b, Reglamento de la Ley Federal sobre Monumentos y Zonas Arqueológicos, Artísticos e Históricos: México, Instituto Nacional de Antropología e Historia.

Instituto Nacional de Antropología e Historia, 2003, Coordinación Nacional de Arqueología, Manual General de Organización, http://paginah. inah.gob.mx/modules.php?name=News\&file=print $\&$ sid=239\&top ico=10. Marzo 2006 .

Instituto Nacional de Antropología e Historia, 2009, Trámites INAH, http://dti.inah.gob.mx/index.php?option=com content\&task=view\&id=2700. Marzo 2009.

Mirambell, L., Polaco, O.J., Sanchéz M.F., 1988, El Instituto Nacional de Antropología e Historia y el patrimonio paleontológico: Antropología, 22, 6-7.

Muñoz-Collazos, M.A., 2006, Gestión participativa del patrimonio: un caso boliviano: Cuadernos de antropología y patrimonio cultural, 4, 1-48.

Nyborg, T., Alvarado-Ortega, J., Blanco, A., Vega, F.J., 2005, Taphonomy of fish preserved within ammonite chambers from the Upper Cretaceous Austin Group, Coahuila, Mexico, en: Geological Society of America, Abstracts with Program, 37, 158.

Schenk, E.T., McMasters, J.M., 1948, Procedure in Taxonomy: California, Stanford University Press.

Secretaría de Programación y Presupuesto (SPP), 1983, Síntesis geográfica de Coahuila: México, Instituto Nacional de Estadística, Geografía e Informática.

Stinnesbeck, W., Ifrim, C., Schmidt, H., Rindfleisch, A., Buchy M.C., Frey, E., González-González, A.H., Vega, F.J., Cavin, L., Séller, G., Smith K.T., 2005, A new lithographic limestone deposit in the Upper Cretaceous Austin Group at El Rosario, county of Múzquiz, Coahuila, northeastern Mexico: Revista Mexicana de Ciencias Geológicas, 22, 401-418.

Vega, F.J., Nyborg, T., Rojas-Briceño, A., Patarroyo, P., Luque, J., PorrasMúzquiz, H., Stinnesbeck, W., 2007, Upper Cretaceous Crustacea from Mexico and Colombia: similar faunas and environments Turing Turonian times: Revista Mexicana de Ciencias Geológicas, 24 (3), 403-422.

Manuscrito recibido: Febrero 27, 2009.

Manuscrito corregido recibido: Junio 1, 2009.

Manuscrito aceptado: Junio 5, 2009. 